\title{
Partial calcaneal decortication and vastus lateralis free muscle flap for chronic recurrent calcaneal osteomyelitis
}

\begin{abstract}
Introduction: Patients with chronic calcaneal osteomyelitis have below knee amputation or total calcanectomy as durable coverage is difficult. The purpose of this report was to present a $39 \mathrm{yr}$ old male with chronic recurrent calcaneal osteomyelitis since $10 \mathrm{yrs}$ treated by saucerisation, partial calcaneal decortication and free vastus lateralis muscle flap.

Method: Pre op evavation was done with x-ray \& CT scan to evalvate the extent of infection, presence of seqestrum and to assess the approximate defect left post saucerization. Calcaneum was approached laterally, initially thorough debridement of the existing ulcer followed by saucerisation \& curettage of calcaneum was done. In the second sitting after the blood markers and xrays were negative for infection the cavity in the calcaneum was filled by a vastus lateralis free muscle flap connecting the lateral circumflex femoral artery to the anterior tibial artery and covered with a skin graft. Ankle was stabilized with external fixator.

Result and Discussion: The muscle flap and the surgical wound healing were remarkable at the end of 6 weeks. The external fixator was removed after a period of 2 months. 8 weeks $\mathrm{x}$-ray showed filling of the defect in calcaneum with callus. By the end of 4 month whole of the calcaneum defect was filled with cancellous bone. Patient was able to walk full weight bearing without pain at the end of 6 months. He had minimal stiffness of foot and ankle movement at the end of 4 years post surgery. In comparison to the previously practiced surgeries of total calcanecomy, partial calcanectomy and Gaenslen's split heel approach for calcaeal osteomyelitis, this technique was found to produce better functional and cosmetic outcomes with limited disability.

Conclusion: 'Free muscle flap provides vascular base for good callus formation and helps in the healing of defect in cases of chronic osteomyelitis of calcaneum. Thorough debridment of infected bone and there should be no signs of infection radiologically or in blood parameters before the muscle flap is performed.
\end{abstract}

\section{Introduction}

Osteomyelitis is a dreaded complication in the field of orthopaedics. Osteomyelitis of the long bone is well known to be associated with open fractures. In Wang et al. ${ }^{1}$ put the number of $\mathrm{CO}$ to 7 to $8 \%$ of all bone infections based on studies of Feigin et al. ${ }^{2}$ In the literature often wound infection, soft tissue infection and bone infection will not be differentiated. Calcaneum osteomyelitis can be post traumatic or based on chronic pressure or accompanied by diabetes. ${ }^{3}$ This if not well treated with adequate debridment could lead to deformity, loss of limb partial or full or risk to life. Patients who have a recurrent discharging sinus of the heel with chronic calcaneal osteomyelitis \& non healing ulcer often have a below-the-knee amputation because durable soft tissue coverage cannot be obtained over the calcaneus. Saucerisation of bone provides a good raw surface for free muscle flap to be inserted by revascularisation procedure. An excellent functional result has been obtained by performing this procedure.

\section{Case report}

\section{History and examination}

In this case report we present a thirty nine year old male who had a chronic and recurrent ostemyelitis of the right calcaneum since ten years due to a thorn injury, who was repeatedly treated with saucerisation.
Volume 9 Issue I - 2017

\section{Bharath Raj R, Vikas Ellur, Prashant Tejwani, Sushil Nahar, Ashok Raj Koul}

Department of Orthopaedics and sports medicine, Ramakrishna Hospital, India

\section{Correspondence: Bharath Raj R, 853B, 26th main, 2nd stage, Ist phase, BTM layout, Bangalore 560076, India, Tel 9686343580,} Email bharathraj.r@gmail.com

Received: September 09, 2016 | Published: September 29 2017
At presentation he had a discharging sinus over the heel with ulcer adjacent to it and the ulcer was fixed to the underlying calcaneum. $\mathrm{X}$-rays (Figures $1 \& 2$ ) were taken pre operatively which showed evidence of lysis of the entire cortex which is indicative of calcaneal osteomyeltis. Seeing the presence of lysis on x-ray, it was assumed the lytic lesion to be small and the patient was taken up for surgery as CT/ MRI was not considered necessary.

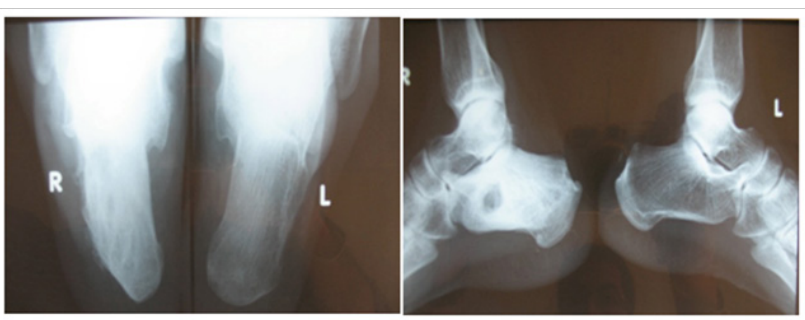

Figure I\&2 Pre operativeX-rays of shows lysis of the entire cortex.

\section{Operative Procedure I}

The patient underwent debridement and saucerisation of the calcaneum. As good raw surface with fresh bleeding was visible from the base of the lytic area as on xray therefore debridement was considered adequate and wound was closed with 1 ethilon and limb was kept elevated in order to reduce the swelling. Fig 
Post op day two showed, active pus discharge from wound site Probablity of multiple pockets or draining sinuses was taken up and MRI of the calcanuem was done (Figures $3 \& 4$ ). This showed multiple pockets of pus collection in the calcaneum.
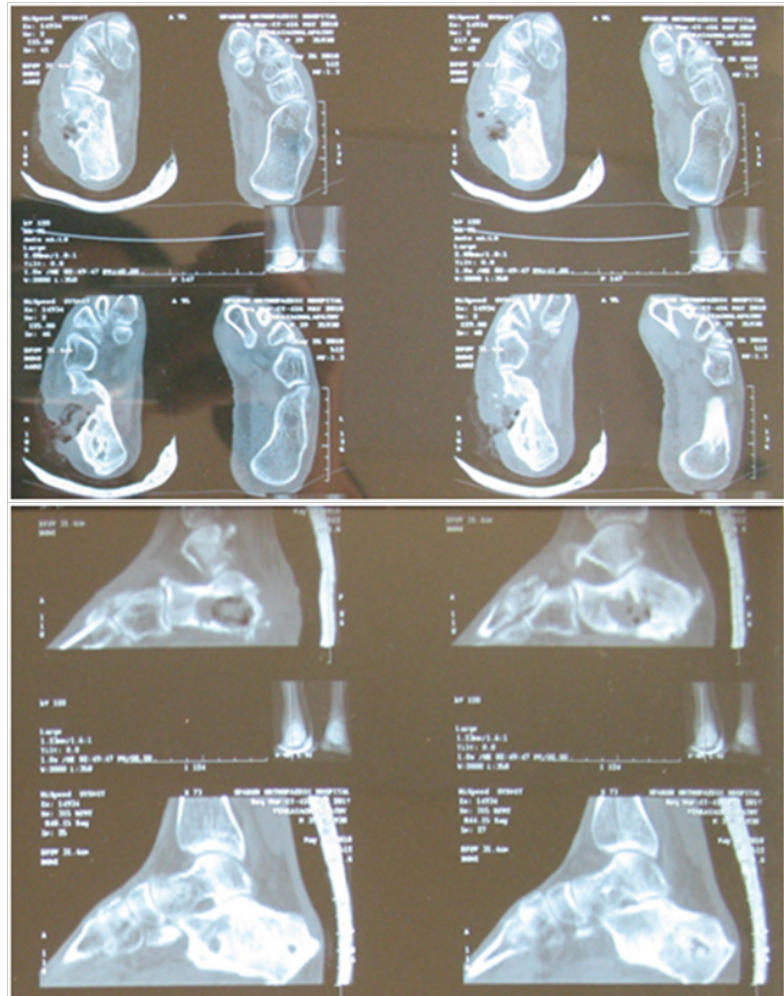

Figure 3\&4 MRI shows multiple pockets of pus collection in the calcaneum.

\section{Operative procedure 2}

The second surgery was planned on post operative day 4. It was planned to do the surgery in two stages. A partial calcaneal decortication of the calcaneum was planned in the first stage where the entire bone was debrided and wound was closed with a drain and vaccum dressing. After a period of five days when the drain was empty and no collection was present, patient was taken up for second stage surgery. The second stage of the surgery involved applying an external fixator to the ankle. The external fixator was applied to allow no ankle movement to happen in order to prevent any stretch of the anastomosis which was going to be done for the free flap. The empty space left in the calcaneum was filled by a vastus lateralis free muscle flap harvested from the contralateral thigh along with its feeding artery the lateral branch of descending circumflex femoral artery. The feeding artery was then anastamosed with the anterior tibial artery. The flap area over the calcaneum was closed with a skin graft. The wound area over the contralateral thigh was closed primarily in multiple layers with 1 vicryl for IT band, 2-0 for subcutaneous and staples for skin with a 14 size drain (Figures 5 \& 6).

\section{Post operative period}

The muscle flap and the surgical wound healing were remarkable at the end of six weeks. Fixator was removed after two months from the date of the second procdure. Six week x-ray showed filling of the defect in calcaneum with callus. By the end of fourth month whole of the calcaneum defect was filled with cancellous bone.

At the end of two years patient had minimal stiffness of ankle joint and was able to walk full weight bearing without any pain and $\mathrm{x}$-ray showed no signs of infection (Figures 7-10). Blood parameters of complete blood count, $\mathrm{C}$ reactive protein and Erythrocyte sedimentation rate were done which were all found to be within normal limits.
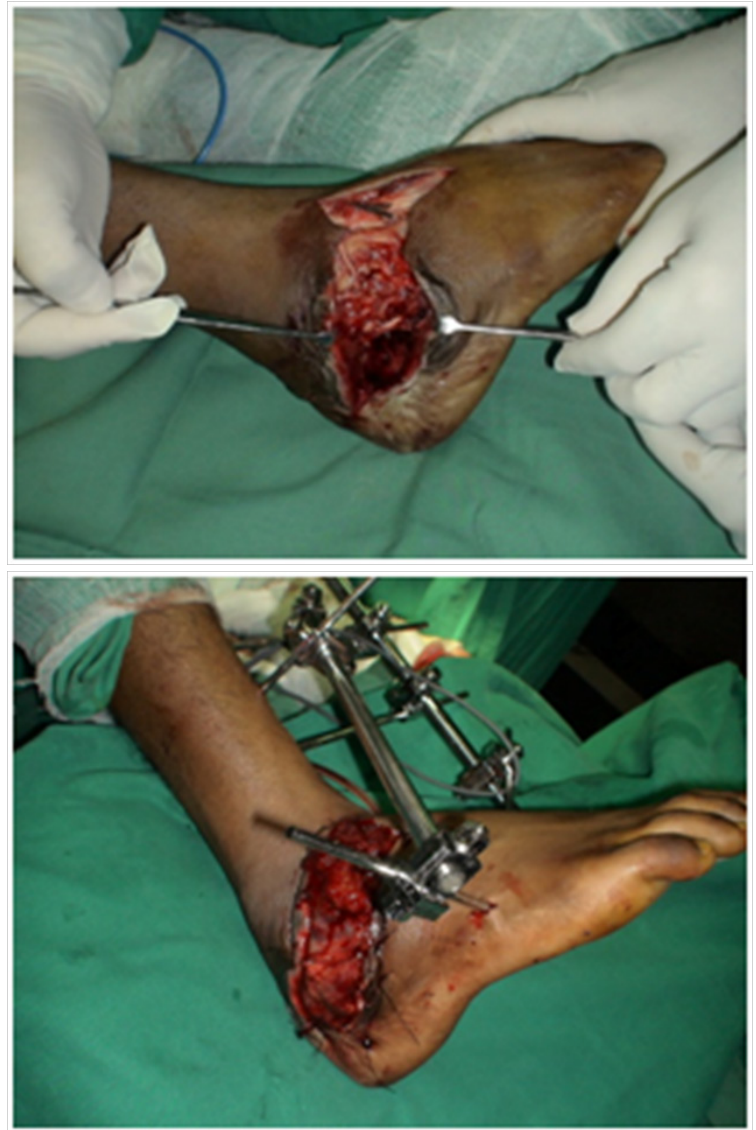

Figure 5\&6 Flap area over the calcaneum was closed with a skin graft.
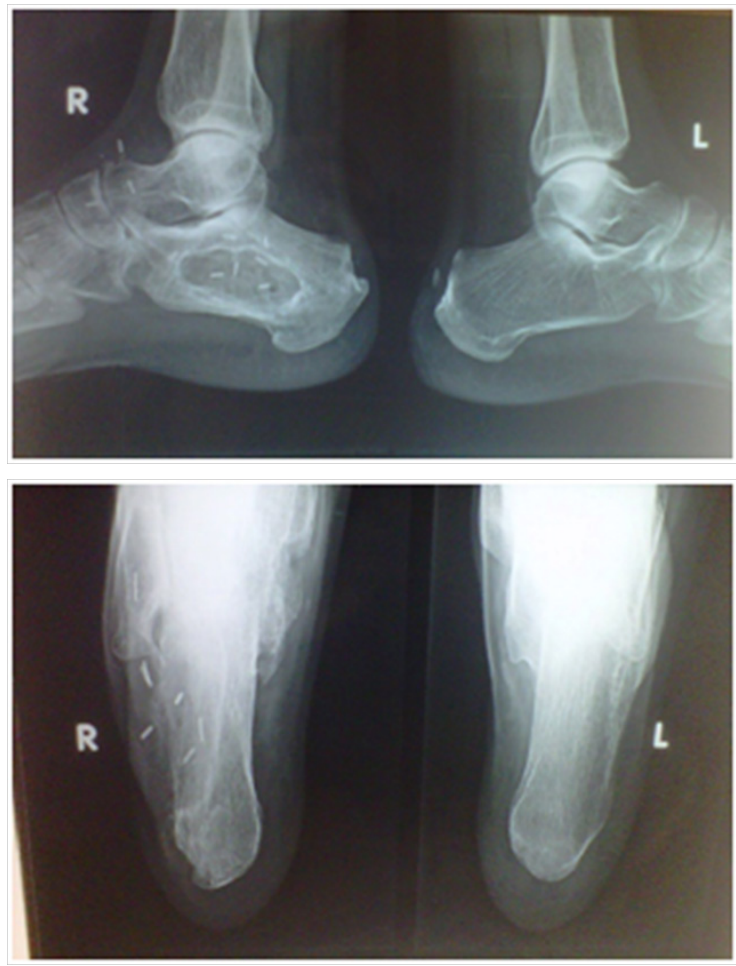

Figure $7 \& 8$ x-ray showed no signs of infection. 

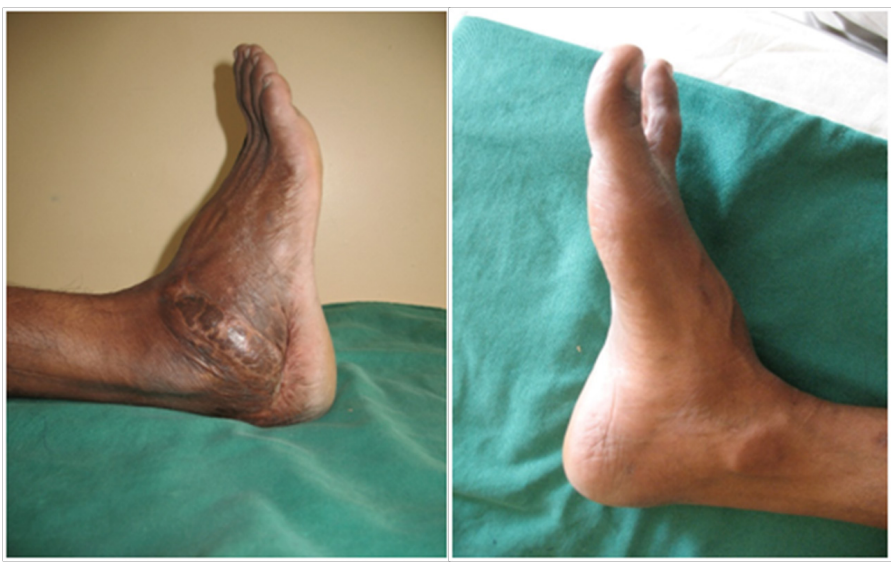

Figure 9\& 10 Minimal stiffness of ankle joint in post operative period.

\section{Discussion}

Calcaneal osteomyelitis has been a challenge to treat, cure and obtain a good functional outcome. The initial method of treatment in chronic recurrent calcaneal osteomyelitis was amputation to prevent further spread of infection proximally and the level of amputation differed for each patient. This was based on the spread and extent of infection in bone and soft tissues.

Partial calcanectomy has been described by many and has been reviewed in detail in the literature. The latest being Cook et al., ${ }^{4}$ who explained the multiple factors affecting the healing outcome of patients with calcaneal osteomyelitis. And this procedure of flap cover ${ }^{5}$ acts as a valid option prior to amputation as it did not compromise the limb and make the patient dependent on a prosthetic. When partial calcanectomy is done, it deforms the calcaneum and causes imbalance in the biomechanics of gait.

Gaenslen $^{6}$ in his initial studies on calcaneal osteomyelitis was of the opinion that wounds were to be left open after debridement and allowed to be healed by secondary intention. By using the split heel approach, adequate drainage can be obtained of the calcaneum and primary closure could be done under tension with the help of antibiotic cover. By this method the patient ends up with a broader heel. The time of union for the split calcaneum ${ }^{7,8}$ was approximately around 12 weeks. The scar if allowed for secondary closure heals with a thick scar which leads to painless extra cushioning while primary closure heals with a thin scar and has chances of break down. This also causes growth disturbance if done in children. By this method, the author gives a chance of re-infection when a thin scar is present as there are higher chances of skin breakdown and with a thick scar it is painless and with decreased sensation increasing the chances of re-infection.
In comparison to that use of a free flap ${ }^{9}$ which can be harvested from the patients own body not only maintains the anatomical architecture of the heel and also gives a better functional outcome of the heel. There are multiple options for a free muscle graft but an anterolateral thigh flap is the easiest to harvest and also ideal for a cavity present in the calcaneum which has no specific shape.

\section{Conclusion}

It is quintessential for a thorough work up of the patient before a surgery is planned. Though the above mentioned procedure requires surgical expertise of both the orthopaedic and plastic team and takes a longer time for recovery, the functional outcome in calcaneal osteomyelitis is good in comparison to the previous mentioned surgeries which have had drawbacks.

\section{Acknowledgments}

None.

\section{Conflicts of interest}

None.

\section{References}

1. Wang EH, Simpson S, Bennet GC. Osteomyelitis of the calcaneum. $J$ Bone Joint Surg Br. 1992;74(6):906-909.

2. Feigin RD, McAlister WH, Joaquin VH, et al. Osteomyelitis of the calcaneus. Report of eight cases. Am J Dis Child. 1970;119(1):61-65.

3. Fukuda T, Reddy V, Ptaszek AJ. The infected calcaneus. Foot Ankle Clin. 2010;15(3):477-486.

4. Cook J, Cook E, Landsman A, et al. A retrospective assessment of partial calcanectomies and factors influencing postoperative course. $J$ Foot Ankle Surg. 2007;46:248-255.

5. Boffeli TJ, Collier RC (2013) Near total calcanectomy with rotational flap closure of large decubitus heel ulceration complicated by calcaneal osteomyelitis. J Foot Ankle Surg 52(1): 107-112.

6. Bhattacharya A, Das R. Gaenslen's Split Heel Approach for the Treatment of Chronic Osteomyelitis of the Calcaneus: A series of three cases. The Foot and Ankle Online Journal. 2010;3(11):3.

7. Tiemann AH, Hofman GO, Steen M,et al. Adult Calcaneal Osteitis: incidence, etiology, diagnostics and therapy. GMS interdisciplinary plastic and reconstructive surgery, $D G P W .2012 ; 1: 1-8$.

8. Wang EHM, Simpson S, Bennet CG. Osteomyelitis of the calcaneum. Journal of bone and joint surgery. 1992;74B(6):906-909.

9. Landyut KV. Anterolateral thigh flap for lower extremity reconstruction. Semin Plast Surg. 2006;20(2):127-132. 